\title{
Effective solution of the $D(-1)$-quadruple conjecture
}

\author{
by
Andrej Dujella (Zagreb), Alan Filipin (Zagreb) and Clemens Fuchs (Zürich)

1. Introduction. Let $n$ be an integer. A set of $m$ positive integers is called a Diophantine $m$-tuple with the property $D(n)$, or simply a $D(n)-m$ tuple, if the product of any two of them increased by $n$ is a perfect square.

A lot of work has been done on $D(n)$ - $m$-tuples in the last decade, but in fact the problem is much older. It was first studied by Diophantus in the case $n=1$. He found a set of four positive rationals with the above property: $\{1 / 16,33 / 16,17 / 4,105 / 16\}$. The first $D(1)$-quadruple however, the set $\{1,3,8,120\}$, was found by Fermat. Later Euler was able to add the fifth positive rational, 777480/8288641, to Fermat's set (see [6], [7, pp. 103-104, 232], [8, pp. 141-145] and [24, pp. 177-181]). Recently, Gibbs [22] found examples of sets of six positive rationals with the property of Diophantus. The folklore conjecture is that there does not exist a $D(1)$-quintuple. In 1969, Baker and Davenport [2] proved that Fermat's set cannot be extended to a $D(1)$-quintuple. Recently, the first author proved that there does not exist a $D(1)$-sextuple and there are only finitely many $D(1)$-quintuples (see [15], this refined previous results $[19,12])$. The last result is effective, namely if $\{a, b, c, d, e\}$ is a $D(1)$-quintuple, then $d<10^{2171}$ and $e<10^{10^{26}}$ (cf. [15, Corollary 4, p. 210]). This proves the $D(1)$-quintuple conjecture in an effective way, leaving open a completely determined finite set to check.

For general $n$, it is easy to see, by considering congruences modulo 4 , that if $n \equiv 2(\bmod 4)$ then there does not exist a $D(n)$-quadruple (see $[5,23,29])$, while the first author proved in [9] that if $n \not \equiv 2(\bmod 4)$ and $n \notin$ $S=\{-4,-3,-1,3,5,8,12,20\}$, then there exists at least one Diophantine quadruple with the property $D(n)$. The conjecture is that for $n \in S$ there does not exist a Diophantine quadruple with the property $D(n)$.

2000 Mathematics Subject Classification: Primary 11D09, 11D45; Secondary 11B37, $11 \mathrm{~J} 68$.

Key words and phrases: Diophantine $m$-tuples, Pell equation, Diophantine approximation. 
The best known upper bounds for the size of sets with the property $D(n)$ are logarithmic in $|n|$ (see $[14,16])$. Moreover, for $n$ prime it was shown recently that the size is bounded by the absolute constant $2^{170}$ (cf. [18]).

Therefore, in the case $n=-1$ the conjecture is that there does not exist a $D(-1)$-quadruple. In analogy to above this is known as the $D(-1)$ quadruple conjecture and it appeared explicitly in [10] for the first time. It is known that some particular $D(-1)$-triples cannot be extended to $D(-1)$ quadruples, namely this was verified for the triples $\{1,2,5\}$ (by Brown in [5], see also $[32,25,31,26]),\{1,5,10\}$ (by Mohanty and Ramasamy in [28]), $\{1,2,145\},\{1,2,4901\},\{1,5,65\},\{1,5,20737\},\{1,10,17\},\{1,26,37\}$ (by Kedlaya [25]) and $\{17,26,85\}$ (again by Brown in [5]). Moreover, Brown proved that the infinite families $\left\{x^{2}+1,(x+1)^{2}+1,(2 x+1)^{2}+4\right\}$, if $x \not \equiv 0$ $(\bmod 4),\left\{2,2 x^{2}+2 x+1,2 x^{2}+6 x+5\right\}$, if $x \equiv 1(\bmod 4)$ of $D(-1)$-triples cannot be extended to quadruples. The first author proved the conjecture in [11] for all triples of the form $\{1,2, c\}$.

Very recently, Dujella and Fuchs [17] proved that there does not exist a $D(-1)$-quintuple. More precisely, they proved that there does not exist a $D(-1)$-quadruple $\{a, b, c, d\}$ with $2 \leq a<b<c<d$. This implies that we are left with the case $a=1$, i.e. $D(-1)$-quadruples of the form $\{1, b, c, d\}$. In [17] it was remarked that the case $a=1$ seems more involved and much harder and that it can be compared with the strong version of the quintuple conjecture for $n=1$, which says that every $D(1)$-triple can be extended to a $D(1)$-quadruple in an essentially unique way.

In the meantime the nonextendability of $\{1, b, c\}$ was confirmed for $b=5$ (in [1]), for $b=10$ (by the second author [20]), and for $b=17,26,37,50$ (by Fujita in [21]).

The aim of the present paper is to go further and to prove that in fact there are at most finitely many $D(-1)$-quadruples $\{1, b, c, d\}$. We will prove the following result:

Theorem 1. Let $\mathcal{D}=\{1, b, c\}$ be a $D(-1)$-triple. Then:

(i) If $c>b^{9}$, then $\mathcal{D}$ cannot be extended to a $D(-1)$-quadruple $\{a, b, c, d\}$ such that $d>c$.

(ii) If $11 b^{6} \leq c \leq b^{9}$, then $\mathcal{D}$ cannot be extended to a $D(-1)$-quadruple. Assume that $\{1, b, c, d\}$ with $1<b<c<d$ is an extension of $\mathcal{D}$ to a $D(-1)$-quadruple.

(iii) If $b^{3} \leq c<11 b^{6}$, then $c<10^{238}, d<10^{10^{23}}$.

(iv) If $b^{1.1} \leq c<b^{3}$, then $c<10^{491}, d<10^{10^{23}}$.

(v) If $3 b<c<b^{1.1}$, then $c<10^{94}, d<10^{10^{21}}$.

(vi) If $c=1+b+2 \sqrt{b-1}$, then $c<10^{74}, d<10^{10^{21}}$. 
Note that, by [17, Lemma 7], if $c>b$ and $c \neq 1+b+2 \sqrt{b-1}$ then it follows that $c>3 b$. Hence, the theorem covers all possible $D(-1)$-triples $\{1, b, c\}$ such that $1<b<c$.

To sum up, we get the following effective solution of the $D(-1)$-quadruple conjecture:

TheOREM 2. There are only finitely many $D(-1)$-quadruples. Moreover, if $\{a, b, c, d\}$ is a $D(-1)$-quadruple, then $\max \{a, b, c, d\}<10^{10^{23}}$.

As in the case for the $D(1)$-quintuple conjecture, this proves the $D(-1)$ quadruple conjecture in an effective way. Unfortunately, the remaining set is too large to be checked by means of a computer program at present.

This is also the first nontrivial result (for integers $\not \equiv 2(\bmod 4))$ related to the following conjecture:

CONJECTURE 1. If $n$ is not a perfect square, then there exist only finitely many $D(n)$-quadruples.

Since, by [9, Remark 3], all elements of a $D(-4)$-quadruple are even, Theorem 2 implies that Conjecture 1 is valid for $n=-1$ and $n=-4$.

The case $n=-1$ deserves special attention because it is closely connected with another old problem investigated by Diophantus and Euler. Namely, Diophantus studied the problem of finding numbers such that the product of any two increased by the sum of these two gives a square. He found two triples $\{4,9,28\}$ and $\{3 / 10,21 / 5,7 / 10\}$ satisfying this property. Euler found a quadruple $\{5 / 2,9 / 56,9 / 224,65 / 224\}$ and asked if there is an integer solution of this problem (see [6], [7, pp. 85-86, 215-217], [8, pp. 101-104] and [24, pp. 162-164, 344-347]). In [13] an infinite family of rational quintuples with this property was given. Since

$$
x y+x+y=(x+1)(y+1)-1,
$$

we see that the problem of finding integer $m$-tuples with the property that for any two distinct elements the product plus their sum is a perfect square is equivalent to finding $D(-1)$ - $m$-tuples. In fact the main result in [17] completely solved this problem, namely it was shown that there does not exist a set of four positive integers such that the product of any two distinct elements plus their sum is a perfect square.

By the equivalence (1), we get the following corollaries to the above theorems, which extend the problem solved in [17].

COROLlary 1. There are at most finitely many sets $\left\{a^{2}, b^{2}, c^{2}\right\}, 0<a<$ $b<c$ of three positive perfect squares such that the product of any two of its distinct elements plus their sum is a perfect square. 
COROLlary 2. There are at most finitely many sets of four integers with the property that the product of any two of its distinct elements plus their sum is a perfect square.

In the next section we will start by collecting useful information about this problem (especially from [17]). The strategy of proof follows the same lines as the proofs of almost all other recent results on nonextendability of $D(n)$-m-tuples. First we reduce the problem of finding $d$ which extends $\{1, b, c\}$ to a $D(-1)$-quadruple to a system of simultaneous Pellian equations, which leads to the consideration of intersections of linear recurring sequences. We know that the indices of these sequences have the same parity. Moreover, we have a very important congruence relation, which e.g. implies that the sequences cannot have intersections for small indices. Moreover, by the gap principle, which was the main improvement in the proof in [17], we get precise information on the initial terms of the recurring sequences.

If $c \geq 11 b^{6}$, in Section 3 we obtain (Proposition 1) by Bennett's theorem on simultaneous approximations of square roots which are close to 1 (in a slightly refined version for our context by Fujita [21]) parts (i) and (ii) (the case of very large solutions) of our main theorem (Theorem 1).

In Section 4 we will compute some general upper bound for the indices of the recurring sequences in terms of $c$ by using Baker's theory of linear forms in logarithms of algebraic numbers (in fact we will use Matveev's result [27]).

In Section 5 we will give the proof of parts (iii)-(v) (large, medium size, and small solutions, respectively) of the theorem (see Propositions 2-4), where we have to get lower bounds for $n$ in terms of some power of $c$. For medium size (part (iv), i.e. $b^{1.1} \leq c<b^{3}$ ) and small (part (v), i.e. $\left.3 b<c<b^{1.1}\right)$ solutions we first have to refine the congruence relations. In fact this is the most important new part of the proof.

Finally, we will give the proof of the case of very small solutions $(c \leq 3 b$, part (vi) of the theorem) in Section 6 (Proposition 5).

2. Preliminaries. Let $\{1, b, c\}$, where $1<b<c$, be a $D(-1)$-triple and let $r, s, t$ be positive integers defined by

$$
b-1=r^{2}, \quad c-1=s^{2}, \quad b c-1=t^{2} .
$$

The symbols $r, s, t$ will always have this meaning. Assume that there is a positive integer $d>c$ such that $\{1, b, c, d\}$ is a $D(-1)$-quadruple. We have

$$
d-1=x^{2}, \quad b d-1=y^{2}, \quad c d-1=z^{2},
$$

with integers $x, y, z$. Eliminating $d$ from (2) we obtain the following system of Pellian equations:

$$
\begin{aligned}
z^{2}-c x^{2} & =c-1 \\
b z^{2}-c y^{2} & =c-b .
\end{aligned}
$$


By combining these two equations we additionally have $y^{2}-b x^{2}=b-1$. We describe the sets of solutions of (3) and (4) in the following lemma.

Lemma 1. If $(z, x)$ and $(z, y)$, with positive integers $x, y, z$, are solutions of (3) and (4) respectively, then there exist integers $z_{0}, x_{0}$ and $z_{1}, y_{1}$ with

(i) $\left(z_{0}, x_{0}\right)$ and $\left(z_{1}, y_{1}\right)$ are solutions of (3) and (4) respectively,

(ii) the following inequalities are satisfied:

$$
\begin{array}{ll}
\left|x_{0}\right|<s, & 0<z_{0}<c, \\
\left|y_{1}\right|<t, & 0<z_{1}<c,
\end{array}
$$

and there exist integers $m, n \geq 0$ such that

$$
\begin{aligned}
& z+x \sqrt{c}=\left(z_{0}+x_{0} \sqrt{c}\right)(s+\sqrt{c})^{2 m} \\
& z \sqrt{b}+y \sqrt{c}=\left(z_{1} \sqrt{b}+y_{1} \sqrt{c}\right)(t+\sqrt{b c})^{2 n} .
\end{aligned}
$$

Proof. This is [17, Lemma 1].

From (7) we conclude that $z=v_{m}$ for some $\left(z_{0}, x_{0}\right)$ with the above properties and integer $m \geq 0$, where

$$
v_{0}=z_{0}, \quad v_{1}=(2 c-1) z_{0}+2 s c x_{0}, \quad v_{m+2}=(4 c-2) v_{m+1}-v_{m} .
$$

Hence for varying $m \geq 0$ the solutions $z$ form a binary recurrent sequence $\left(v_{m}\right)_{m \geq 0}$ whose initial terms are found by solving equation (7) for $z$ when $m=0$ and 1 , and whose characteristic equation has the roots $(s+\sqrt{c})^{2}$ and $(s-\sqrt{c})^{2}$. In the same manner, from (8), we conclude that $z=w_{n}$ for some $\left(z_{1}, y_{1}\right)$ with the above properties and integer $n \geq 0$, where

$$
w_{0}=z_{1}, \quad w_{1}=(2 b c-1) z_{1}+2 t c y_{1}, \quad w_{n+2}=(4 b c-2) w_{n+1}-w_{n} .
$$

Our system of equations (3) and (4) is thus transformed to finitely many equations of the form $z=v_{m}=w_{n}$.

From (9) and (10) we get by induction

$$
\begin{aligned}
& v_{m} \equiv(-1)^{m} z_{0}(\bmod 2 c), \\
& w_{n} \equiv(-1)^{n} z_{1}(\bmod 2 c), \\
& v_{m} \equiv(-1)^{m}\left(z_{0}-2 c m^{2} z_{0}-2 c s m x_{0}\right)\left(\bmod 8 c^{2}\right), \\
& w_{n} \equiv(-1)^{n}\left(z_{1}-2 b c n^{2} z_{1}-2 c t n y_{1}\right)\left(\bmod 8 c^{2}\right)
\end{aligned}
$$

(see [17, Lemma 2]). So if the equation $v_{m}=w_{n}$ has a solution, then $z_{0}=z_{1}$. Moreover, we have the following properties:

Lemma 2. If $v_{m}=w_{n}, n \neq 0$, then

(i) $m \equiv n(\bmod 2)$,

(ii) $n \leq m \leq 2 n$,

(iii) $m^{2} z_{0}+s m x_{0} \equiv b n^{2} z_{1}+t n y_{1}(\bmod 4 c)$. 
Proof. Parts (i) and (iii) follow at once from $z_{0}=z_{1}$ and the above congruence relations. Statement (ii) is [17, Lemma 5]. Observe that it was remarked in [17, Section 3] that this statement holds for arbitrary $D(-1)$ quadruples and not only in the special situation considered there.

Moreover, we have the following bounds for $v_{m}, w_{n}$, respectively.

LEMma 3. We have

$$
\begin{array}{ll}
(c-1)(4 c-3)^{m-1}<v_{m}<4 c^{2}(4 c-2)^{m-1} & \text { for } m \geq 1, \\
(c-b)(4 b c-3)^{n-1}<w_{n}<4 b c^{2}(4 b c-2)^{n-1} & \text { for } n \geq 1 .
\end{array}
$$

Proof. This is part of the proof of [17, Lemma 5] (cf. (3.1) in [17] and the equation just before that).

We collect some useful gap principles for the elements of $D(-1)$-triples and quadruples.

Lemma 4. Let $\{a, b, c\}$ be a $D(-1)$-triple and $0<a<b<c$. Then $c=a+b+2$ or $c>3 a b \geq 3 b$.

Let $\{1, b, c, d\}$ be a $D(-1)$-quadruple and $1<b<c<d$. Then $d>c^{9}$.

Proof. The first part of the lemma is [17, Lemma 7]. The second part is a consequence of [17, Lemma 13]. Observe that in fact it is proved there for the $D(-1)$-quadruple $\{1, a, b, c\}$ with $1<a<b<c$. The assumption that $\{a, b, c, d\}$ with $2 \leq a<b<c<d$ and $d$ minimal among all such quadruples was only needed to ensure that $\{1, a, b, c\}$ is really a $D(-1)$-quadruple again (by the use of [17, Lemma 3]).

Lemma 4 has the following important implication on the fundamental solutions of (9) and (10) described in Lemma 1.

Lemma 5. Let the integers $z_{0}, z_{1}, x_{0}, y_{1}$ be as in Lemma 1 . If $c \leq b^{9}$, then

$$
z_{0}=z_{1}=s, \quad x_{0}=0, \quad y_{1}= \pm \sqrt{b-1}= \pm r .
$$

Proof. Set

$$
d_{0}=\frac{z_{0}^{2}+1}{c}
$$

It follows that

$$
d_{0}-1=x_{0}^{2}, \quad b d_{0}-1=y_{1}^{2}, \quad c d_{0}-1=z_{0}^{2}
$$

and $d_{0}<c$ (this construction was the key lemma in [17], in fact it was [17, Lemma 3]). If $d_{0}>1$, then $\left\{1, b, d_{0}, c\right\}$ is again a $D(-1)$-quadruple and by the second part of Lemma 4 it follows that $c>b^{9}$, a contradiction. Hence, $d_{0}=1$, which implies our assertion (cf. also [17, Lemma 4] for this final conclusion). 
It is easy to show that the equation $v_{m}=w_{n}$ cannot have solutions with small indices.

LEMma 6. We have $v_{1} \neq w_{1}, v_{2} \neq w_{2}, v_{4} \neq w_{2}$.

Proof. See [17, Lemmas 8, 10 and 11]. Observe again that the conclusions of these lemmas are independent of the existence of a minimal extension, which was assumed there.

We have collected all information we need to prove our main theorem, which will be done in the following sections.

3. Very large solutions. The proof will follow from a lower bound for $m, n$ in terms of some power of $c$, together with a logarithmic upper bound obtained from Bennett's theorem. The idea for obtaining the lower bound is that if $v_{m}=w_{n}$, then we can study the congruence from Lemma 2(iii). We will show that if $b, m, n$ are small compared with $c$, these congruences are equalities which contradict the equality $v_{m}=w_{n}$.

LEMMA 7. If $v_{m}=w_{n}, n \neq 0,1$ and $c \geq 11 b^{6}$, then $n>c^{1 / 6}$.

Proof. From Lemma 2(iii) we have $m^{2} z_{0}+s m x_{0} \equiv b n^{2} z_{0}+t n y_{1}(\bmod 4 c)$. Assume now that $n \leq c^{1 / 6}$. It is easy to see that $\max \left\{\left|m^{2} z_{0}\right|,\left|s m x_{0}\right|,\left|t n y_{1}\right|\right\}$ $<\left|b n^{2} z_{0}\right|$. We first consider the case $c>b^{9}$. Let $d_{0}$ be such that $c d_{0}-1=z_{0}^{2}$. If $d_{0}>1$ then $\left\{1, b, d_{0}, c\right\}$ is a $D(-1)$-quadruple and we see by the second part of Lemma 4 that $c>\left(\max \left\{b, d_{0}\right\}\right)^{9}>b^{6} d_{0}^{3}$. Then we have

$$
\left|b n^{2} z_{0}\right|<b \cdot c^{1 / 3} \cdot\left(c d_{0}\right)^{1 / 2}<c^{1 / 3} \cdot c^{1 / 2} \cdot\left(b^{6} d_{0}^{3}\right)^{1 / 6}<c .
$$

If $d_{0}=1$ then we find as in the proof of Lemma 5 that $z_{0}=s$. Thus we immediately get

$$
\left|b n^{2} z_{0}\right|<c^{1 / 9} \cdot c^{1 / 3} \cdot c^{1 / 2}<c .
$$

Now we consider the case $11 b^{6} \leq c \leq b^{9}$. Here we can use Lemma 5 to get

$$
\left|b n^{2} z_{0}\right|<c^{1 / 6} \cdot c^{1 / 3} \cdot c^{1 / 2}=c .
$$

Therefore we conclude that $m^{2} z_{0}+s m x_{0}=b n^{2} z_{0}+t n y_{1}$. We have

and

$$
m^{2} z_{0}+s m x_{0}<m^{2} \sqrt{c d_{0}}+m \sqrt{c d_{0}}<m(m+1) \sqrt{\frac{c}{b}} \sqrt{\left|y_{1}\right|^{2}+1}
$$

$b n^{2} z_{0}-t n\left|y_{1}\right|>\sqrt{b c}\left|y_{1}\right| n^{2}-t n\left|y_{1}\right|>\sqrt{b c}\left|y_{1}\right| n^{2}-\sqrt{b c}\left|y_{1}\right| n=\sqrt{b c}\left|y_{1}\right| n(n-1)$.

This implies

$$
m(m+1) \sqrt{\left|y_{1}\right|^{2}+1}>b\left|y_{1}\right| n(n-1)
$$

and so, as $m \leq 2 n$ and $b \geq 65$, we get

$$
5 \geq \frac{2 n+1}{n-1}>\frac{65\left|y_{1}\right|}{2 \sqrt{\left|y_{1}\right|^{2}+1}}>\frac{65}{2 \sqrt{2}} .
$$

This contradiction proves the statement. 
Next we need an upper bound for $n$ in terms of $c$. To obtain it we use the following slightly modified version of a special case of Bennett's theorem [4] (it is also a modified version of Rickert's theorem [30]), which is Theorem 3.5 in $[21]$.

Lemma 8. Let $b$ and $N$ be integers with $b \geq 5$ and $N \geq 2.39 b^{7}$. Then the numbers

$$
\theta_{1}=\sqrt{1+\frac{1-b}{N}} \text { and } \theta_{2}=\sqrt{1+\frac{1}{N}}
$$

satisfy

$$
\max \left\{\left|\theta_{1}-\frac{p_{1}}{q}\right|,\left|\theta_{2}-\frac{p_{2}}{q}\right|\right\}>\left[32.1 \frac{b^{2}(b-1)^{2}}{2 b-1} N\right]^{-1} q^{-1-\lambda}
$$

for all integers $p_{1}, p_{2}, q$ with $q>0$, where

$$
\lambda=\frac{\log \frac{16.1 b^{2}(b-1)^{2} N}{2 b-1}}{\log \frac{3.37 N^{2}}{b^{2}(b-1)^{2}}}<1 .
$$

We apply this theorem with $N=t^{2}=b c-1 \geq 11 b^{7}-1>2.39 b^{7}$, $p_{1}=b s x, p_{2}=b z, q=t y$, i.e.

$$
\theta_{1}=\frac{s \sqrt{b}}{t}, \quad \theta_{2}=\frac{\sqrt{b c}}{t} .
$$

First we show that the solutions of our problem induce good approximations to the roots $\theta_{1}, \theta_{2}$.

Lemma 9. All positive integer solutions $x, y, z$ of (3) and (4) satisfy

$$
\max \left\{\left|\theta_{1}-\frac{b s x}{t y}\right|,\left|\theta_{2}-\frac{b z}{t y}\right|\right\}<\frac{b-1}{y^{2}} .
$$

Proof. Since $f(x)=b(x-1) /(b x-1)$ is an increasing function we get

$$
\left|\theta_{1}-\frac{b s x}{t y}\right|=\frac{s \sqrt{b}}{t}\left|1-\frac{x \sqrt{b}}{y}\right|=\frac{s \sqrt{b}}{t}\left|1-\frac{b x^{2}}{y^{2}}\right|\left|1+\frac{x \sqrt{b}}{y}\right|^{-1}<\frac{b-1}{y^{2}} .
$$

Moreover, since $b z / y>\sqrt{b c}$ we have

$$
\begin{aligned}
\left|\theta_{2}-\frac{b z}{t y}\right| & =\frac{1}{t}\left|\sqrt{b c}-\frac{b z}{y}\right|=\frac{b}{t}\left|c-\frac{b z^{2}}{y^{2}}\right|\left|\sqrt{b c}+\frac{b z}{y}\right|^{-1} \\
& <\frac{b}{t} \frac{c-b}{y^{2}} \frac{1}{2 \sqrt{b c}}<\frac{1}{2 y^{2}} \frac{b c-1}{\sqrt{b c(b c-1)}}<\frac{b-1}{y^{2}}
\end{aligned}
$$

Now we are ready to prove the first two parts of Theorem 1 . Namely, we show the following proposition. 
Proposition 1. Let $\{1, b, c\}$ with $1<b<c$ be a $D(-1)$-triple. If $c \geq 11 b^{6}$, then there does not exist an integer $d>c$ such that $\{1, b, c, d\}$ is a $D(-1)$-quadruple. If $11 b^{6} \leq c \leq b^{9}$, then the triple $\{1, b, c\}$ cannot be extended to a $D(-1)$-quadruple.

Proof. Combining the upper bound of Lemma 9 with the lower bound from Lemma 8 we get

$$
\left[32.1 \frac{b^{2}(b-1)^{2}}{2 b-1} t^{2}\right]^{-1}(t y)^{-1-\lambda}<\frac{b-1}{y^{2}},
$$

which implies

$$
y^{1-\lambda}<32.1 \frac{b^{2}(b-1)^{3}}{2 b-1} t^{3+\lambda}<\frac{32.1 b^{2}(b-1)^{3}(b c-1)^{2}}{2 b-1} .
$$

We have

$$
\frac{1}{1-\lambda}=\frac{\log \frac{3.37(b c-1)^{2}}{b^{2}(b-1)^{2}}}{\log \frac{3.37(2 b-1)(b c-1)}{16.1 b^{4}(b-1)^{4}}}
$$

which leads to

$$
\log y<\frac{\log \frac{3.37(b c-1)^{2}}{b^{2}(b-1)^{2}} \log \frac{32.1 b^{2}(b-1)^{3}(b c-1)^{2}}{2 b-1}}{\log \frac{3.37(2 b-1)(b c-1)}{16.1 b^{4}(b-1)^{4}}} .
$$

We have (observe that $c \geq 11 b^{6}$ and $b \geq 65, c \geq 82$ )

$$
\begin{aligned}
& \log \frac{3.37(b c-1)^{2}}{b^{2}(b-1)^{2}}<\log \frac{3.37 b^{2} c^{2}}{b^{2}(b-1)^{2}}<2 \log \frac{1.9 c}{b-1}<2 \log (0.03 c), \\
& \log \frac{32.1 b^{2}(b-1)^{3}(b c-1)^{2}}{2 b-1}<\log \left(16.18 b^{6} c^{2}\right)<3 \log (1.14 c), \\
& \log \frac{3.36(2 b-1)(b c-1)}{16.1 b^{4}(b-1)^{4}}>\log \frac{2.29\left(2 b^{2} c-b c-2 b+1\right)}{b^{2} c}>1.51 .
\end{aligned}
$$

Consequently,

$$
\log y<3.98 \log (0.03 c) \log (1.14 c) .
$$

Moreover, from (4) and the first part of Lemma 3, we get

$c y^{2}=b z^{2}-c+b>b(c-1)^{2}(4 c-3)^{2 m-2}-c>b c(4 c-3)^{2 m-2}-c>c(4 c-3)^{2 m-2}$ and therefore $y>(4 c-3)^{m-1}$.

Assume that $n \neq 0,1$. Then by Lemmas 2 and 7 we have $m \geq n>c^{1 / 6}$, which implies

$$
\left(c^{1 / 6}-1\right) \log (4 c-3)<3.98 \log (0.03 c) \log (1.14 c) .
$$


It is easy to check that the function

$$
f(c)=3.98 \log (0.03 c) \log (1.14 c)-\left(c^{1 / 6}-1\right) \log (4 c-3)
$$

is decreasing for $c>0.55 \cdot 10^{12}$. Since $c \geq 11 b^{6} \geq 11 \cdot 65^{6}>0.82 \cdot 10^{12}$, inequality (11) cannot by fulfilled. Hence, $n=0$ or $n=1$. Now, by Lemma $6, z=v_{0}=w_{0}$. But then $d=\left(z^{2}+1\right) / c<c$. Moreover, if $11 b^{6} \leq c \leq b^{9}$, then Lemma 5 implies that $z=s$ and $d=1$. This proves the proposition.

4. A Diophantine approximation result. All other parts of Theorem 1 will be obtained by applying the theory of linear forms in logarithms of algebraic numbers (see [3]) instead of Bennett's approximation result. In this way it will not be possible to exclude any solution, but we will get effective upper bounds. In this section we will prepare the approximation tools that will be used for solutions that are not very large.

In fact we will use a result by Matveev [27], which we quote in a suitable simplified version.

Lemma 10. Let $\Lambda$ be a linear form in logarithms of $l$ multiplicatively independent totally real algebraic numbers $\alpha_{1}, \ldots, \alpha_{l}$ with rational integer coefficients $b_{1}, \ldots, b_{l}\left(b_{l} \neq 0\right)$. Let $h\left(\alpha_{j}\right)$ denote the absolute logarithmic height of $\alpha_{j}, 1 \leq j \leq l$. Define $D=\left[\mathbb{Q}\left(\alpha_{1}, \ldots, \alpha_{l}\right): \mathbb{Q}\right], A_{j}=\max \left\{D h\left(\alpha_{j}\right),\left|\log \alpha_{j}\right|\right\}$ for $1 \leq j \leq l$, and

$$
B=\max \left\{1, \max \left\{\frac{\left|b_{j}\right| A_{j}}{A_{l}}: 1 \leq j \leq l\right\}\right\} .
$$

Then

$$
\log \Lambda>-C(l) C_{0} W_{0} D^{2} \Omega,
$$

where

$$
\begin{array}{cc}
C(l)=\frac{8}{(l-1) !}(l+2)(2 l+3)(4 e(l+1))^{l+1}, \quad W_{0}=\log (1.5 e B D \log (e D)), \\
C_{0}=\log \left(e^{4.4 l+7} l^{5.5} D^{2} \log (e D)\right), \quad \Omega=A_{1} \cdots A_{l} .
\end{array}
$$

Proof. See [27, Theorem 2.1].

Solving the recurrences (3) and (4) we get

$$
\begin{aligned}
& v_{m}=\frac{s}{2}\left[(s+\sqrt{c})^{2 m}+(s-\sqrt{c})^{2 m}\right], \\
& w_{n}=\frac{s \sqrt{b} \pm r \sqrt{c}}{2 \sqrt{b}}\left[(t+\sqrt{b c})^{2 n}+(t-\sqrt{b c})^{2 n}\right] .
\end{aligned}
$$

We now turn the equation $v_{m}=w_{n}$ into an inequality for a linear form in three logarithms, to which Lemma 10 will be applied. 
LEMMA 11. If $v_{m}=w_{n}, n \neq 0$, then

$$
0<2 n \log (t+\sqrt{b c})-2 m \log (s+\sqrt{c})+\log \frac{s \sqrt{b} \pm r \sqrt{c}}{2 \sqrt{b}}<(3.96 b c)^{-n+1} \text {. }
$$

Proof. Let

$$
P=s(s+\sqrt{c})^{2 m}, \quad Q=\frac{s \sqrt{b} \pm r \sqrt{c}}{\sqrt{b}}(t+\sqrt{b c})^{2 n} .
$$

Then $v_{m}=w_{n}$ implies $P+(c-1) P^{-1}=Q+\frac{c-b}{b} Q^{-1}$. Furthermore,

$$
\begin{aligned}
& P>4 s(c-1)=4 \sqrt{c-1}(c-1)>c-1 \\
& Q>\frac{s \sqrt{b}-r \sqrt{c}}{\sqrt{b}}(2 b c-1+2 \sqrt{b c}) \geq 2 c \geq 2 .
\end{aligned}
$$

We get

$$
\begin{aligned}
P-Q & =\frac{c-b}{b} Q^{-1}-(c-1) P^{-1}<(c-1) Q^{-1}-(c-1) P^{-1} \\
& =(c-1)(P-Q) P^{-1} Q^{-1}
\end{aligned}
$$

and therefore $Q>P$. Moreover, $P>Q-(c-1) P^{-1}>Q-1$, which implies that $(Q-P) / Q<Q^{-1} \leq 1 / 2$. Hence,

$$
0<\log \frac{Q}{P}=-\log \frac{P}{Q}=-\log \left(1-\frac{Q-P}{Q}\right)<\frac{Q-P}{Q}+\left(\frac{Q-P}{Q}\right)^{2}
$$

From this we conclude that

$$
\begin{aligned}
0 & <\log \frac{Q}{P}<\frac{1}{Q}+\frac{1}{Q^{2}}<\frac{2}{Q}=\frac{2 \sqrt{b}}{s \sqrt{b} \pm r \sqrt{c}}(t+\sqrt{b c})^{-2 n} \\
& <\frac{2 \sqrt{b}}{s \sqrt{b}-r \sqrt{c}}(3.96 b c)^{-n}=\frac{2 \sqrt{b}(s \sqrt{b}+r \sqrt{c})}{c-b}(3.96 b c)^{-n} \\
& <\frac{4 b \sqrt{c}}{c-b}(3.96 b c)^{-n}<(3.96 b c)^{-n+1} .
\end{aligned}
$$

This proves the lemma.

We use this result to prove a principle to obtain an upper bound for $n$ in all remaining cases.

LEMMA 12. If $v_{m}=w_{n}$, then

$$
\frac{n-1}{\log \frac{160.36 n \log (2.02 b c)}{\log c}}<0.33 \cdot 10^{12} \cdot \log c \cdot \log (b c) .
$$


Proof. We have $l=3, D=4$ and therefore $C(3) \leq 0.65 \cdot 10^{9}$ and $C_{0} \leq 29.89$. Moreover, $\alpha_{1}=s+\sqrt{c}, \alpha_{2}=t+\sqrt{b c}$, and

$$
\alpha_{3}=\frac{s \sqrt{b} \pm r \sqrt{c}}{s \sqrt{b}} .
$$

The numbers $\alpha_{1}, \alpha_{2}, \alpha_{3}$ satisfy the algebraic relations $\alpha_{1}^{2}+(4 c-2) \alpha_{1}+1=0$, $\alpha_{2}^{2}+(4 b c-2) \alpha_{2}+1=0$, and $b(c-1) \alpha_{3}^{2}-2 b(c-1) \alpha_{3}+c-b=0$. Therefore, $A_{1}=2 \log (s+\sqrt{c})<2 \log (2 \sqrt{c})<1.08 \log c, A_{2}=2 \log (t+\sqrt{b c})<$ $\log (\sqrt{2} b c)<1.05 \log (b c)$, and

$$
A_{3} \leq 2 \log \left(b c \frac{s \sqrt{b}+r \sqrt{c}}{s \sqrt{b}}\right)<2 \log (2.02 b c)<2 \log (3.96 b c) .
$$

We also have

$$
A_{3} \geq \log (b(c-1)) \geq \log (64.2 c)>1.95 \log c .
$$

Thus,

$$
B \leq \frac{4 m \log (2.02 b c)}{1.95 \log c} \leq \frac{2.06 m \log (2.02 b c)}{\log c} .
$$

By Lemmas 11 and 10, we therefore get

$$
n-1<0.33 \cdot 10^{12} \cdot \log \frac{80.18 m \log (2.02 b c)}{\log c} \log c \log (b c) .
$$

Finally,

$$
\frac{n-1}{\log \frac{160.36 n \log (2.02 b c)}{\log c}}<0.33 \cdot 10^{12} \cdot \log c \cdot \log (b c) .
$$

Our remaining goal is to calculate a lower bound for $n$ in terms of small powers of $c$ as above. Together with our gap conditions between $b$ and $c$, Lemma 12 will enable us to get effective upper bounds for $c$ in each case. This will be done in the next sections.

5. Large, medium and small solutions. We now start with the remaining cases in Theorem 1. Observe that we always have $c \leq 11 b^{6}$ and therefore, by Lemma $5, z_{0}=z_{1}=s, x_{0}=0, y_{1}= \pm r$.

We start with the case of large solutions, i.e. with $b^{3} \leq c<11 b^{6}$. We have the following lower bound for $n$.

Lemma 13. If $v_{m}=w_{n}, n \neq 0,1$ and $b^{3} \leq c<11 b^{6}$, then $n>c^{1 / 12}$.

Proof. We again start with the relation in Lemma 2(iii), which reads here $m^{2} s \equiv b n^{2} s \pm t n r(\bmod 4 c)$. Assume that $n \leq c^{1 / 12}$. Since $\max \left\{m^{2} s, t n r\right\}$ $<b n^{2} s$, and

$$
b n^{2} s<c^{1 / 3} \cdot c^{1 / 6} \cdot c^{1 / 2}=c,
$$


we have equality in the congruence above, thus $m^{2} s=b n^{2} s \pm t n r$. This implies $4 n^{2} s>n(b s n-t r)$ and therefore

$$
\frac{b \sqrt{c}}{2} n<(b-4) s n<t r<b \sqrt{c},
$$

which gives a contradiction.

Combining the previous lemma with the result from Baker's theory from Lemma 12, we get part (iii) of Theorem 1.

Proposition 2. Let $\{1, b, c, d\}$ with $1<b<c<d$ be a $D(-1)$ quadruple. If $b^{3} \leq c<11 b^{6}$, then $c<0.22 \cdot 10^{238}$ and $d<10^{10^{23}}$.

Proof. Since $b^{3} \leq c$, we get $\log (2.02 b c)<1.50 \log c$, and therefore it follows by Lemmas 12 and 13 that

$$
\frac{n-1}{\log (240.54 n)}<0.29 \cdot 10^{13} \cdot \frac{4}{3}(\log c)^{2}<0.56 \cdot 10^{15}(\log n)^{2} .
$$

Hence, $n<0.60 \cdot 10^{20}$ and therefore $c<0.22 \cdot 10^{238}$.

From Lemma 3 we get $z=v_{m}<4 c^{2}(4 c-2)^{m-1} \leq 4^{m} c^{m+1}$. Consequently,

$$
d \leq \frac{z^{2}+1}{c} \leq \frac{4^{2 m} c^{2 m+2}}{c}=16^{m} c^{2 m+1} .
$$

Thus, $\log _{10} d \leq m \log _{10} 16+(2 m+1) \log _{10} c \leq 0.58 \cdot 10^{23}$ and therefore $d \leq 10^{10^{23}}$.

Next we handle the case of medium size solutions, i.e. with $b^{1.1} \leq c<b^{3}$, and thus prove part (iv) of Theorem 1.

LEMMA 14. If $v_{m}=w_{n}, n \neq 0,1, b^{1.1} \leq c<b^{3}$, and $c>10^{100}$, then $n \geq c^{0.04}$.

Proof. We will consider the congruence of Lemma 2(iii). We have

$$
s\left(m^{2}-b n^{2}\right) \equiv \pm r t n(\bmod 4 c)
$$

Set

$$
A=2 b c-2 r s t-c, \quad B=2 b c+2 r s t-c .
$$

Then $A B=c^{2}+4 b^{2} c-4 b-4 c+4$. Clearly, $B<4 b c$ and $A>\frac{4 b^{2} c}{4 b c}=b$. On the other hand, $A<\frac{c^{2}+4 b^{2} c}{2 b c}<c$. This implies that $2 r s t>2 b c-2 c$, $B>4 b c-3 c$ and

$$
A<\frac{c^{2}+4 b^{2} c}{4 b c-3 c}<\frac{c}{3 b}+\frac{4}{3} b<0.334 c^{0.67}+1.334 c^{0.91}<c^{0.92},
$$

which shows that $A$ is small compared with $c$. Observe that here we use the assumption $c>10^{100}$.

Multiplying both sides of (13) by $2 s$, we get

$$
2\left(m^{2}-b n^{2}\right) \equiv \mp A n(\bmod c) \text {. }
$$


Assume now that $n \neq 0$ and $n<c^{0.04}$. Then $A n<c$ and $\left|2\left(m^{2}-b n^{2}\right)\right| \leq$ $2 b n^{2}<2 c^{0.91+0.08}<c$. Hence, we have equality:

$$
2 b n^{2}-2 m^{2}=A n .
$$

The relation (14) and the inequalities $n \leq m \leq 2 n$ (cf. Lemma 2(ii)) imply

$$
2(b-4) n \leq A \leq 2(b-1) n .
$$

Define $\beta$ by

$$
c=4 b^{2}(2 n-1)+\beta .
$$

We claim that $|\beta|<c^{0.56}$.

Let us first estimate the quantity $\gamma$ defined by $\gamma=4 b A-c-4 b^{2}$. From $A B=c^{2}+4 b^{2} c-4 b-4 c+4$ and $4 b c-3 c<B<4 b c$, we obtain

$$
\begin{aligned}
& \gamma>\frac{1}{4 b c}\left(-16 b^{2}-16 b c+16 b\right)>-8 \\
& \gamma<\frac{3 c+12 b^{2}}{3 b}=\frac{c}{b}+4 b .
\end{aligned}
$$

Therefore, $|\gamma|<c^{0.92}$. Furthermore, from (15) we have $4 b A=8 b^{2} n+\delta$, where $|\delta| \leq 32 b n<c^{0.966}$. Since $\beta=\delta-\gamma$, by putting these estimates together we get

$$
|\beta|<c^{0.966}+c^{0.92}<2 c^{0.966}<c^{0.97} .
$$

Hence,

$$
c=4 b^{2}(2 n-1)+\beta, \quad|\beta|<c^{0.97} .
$$

Analyzing (16) we will improve this estimate for $|\beta|$. Namely, (16) clearly implies $4 b^{2}<c<16 n b^{2}$. It follows that $|\gamma|<c / b+4 b<20 b n<10 n \sqrt{c}$ $<c^{0.55}$, and also $|\delta|<32 b n<c^{0.553}$. Hence, $|\beta|<c^{0.56}$, as claimed.

Now we are ready to finish our argument. By squaring (14), we get

$$
4\left(b n^{2}-m^{2}\right)^{2} \equiv 4(b-1) n^{2}(\bmod c),
$$

which implies

$$
-n^{4} \beta \equiv(2 n-1)\left(4(b-1) n^{2}-4 m^{4}+8 b m^{2} n^{2}\right)(\bmod c) .
$$

But the absolute values of both sides of (17) are less than $c^{0.72}$. Hence, we have equality in (17), and this implies (assuming that $n \neq 0$ )

$$
n^{2} c=(2 n-1)\left(A^{2}-4(b-1)\right) .
$$

Since $A^{2} \equiv 4(b-1)(\bmod c)$, we find that $(2 n-1) \mid n^{2}$, which is possible only if $n=0$ or $n=1$. We obtain a contradiction, proving that $n \geq c^{0.04}$.

Combining this lower bound with the upper bound which follows from Lemma 12, we will again derive that $c$ is bounded by an absolute constant.

Proposition 3. Let $\{1, b, c, d\}$ with $1<b<c<d$ be a $D(-1)$ quadruple. If $b^{1.1} \leq c<b^{3}$, then $c<0.39 \cdot 10^{491}$ and $d<10^{10^{23}}$. 
Proof. Since $b \leq c^{0.91}$, we get $\log (2.02 b c) \leq 1.93 \log c$ and $\log (b c) \leq$ $1.91 \log c$. Now, by Lemmas 14 and 12 ,

$$
\frac{n-1}{\log (309.50 n)}<0.40 \cdot 10^{15}(\log n)^{2} .
$$

Hence, $n<0.42 \cdot 10^{20}$ and thus $c<0.39 \cdot 10^{491}$.

As in the proof of Proposition 2, Lemma 3 implies that $\log _{10} d \leq$ $2 n \log _{10} 16+(4 n+1) \log _{10} c \leq 0.83 \cdot 10^{23}$ and therefore $d \leq 10^{10^{23}}$.

Finally, we turn to part (v) of Theorem 1. This is the case of small solutions, i.e. with $3 b<c<b^{1.1}$.

To get the lower bounds for $m, n$ in terms of some small power of $c$ in the case of small solutions, we will use the following very useful construction, which in fact is the essence of the gap principle in Lemma 4.

Lemma 15. Let $\{a, b, c\}$ be a $D(-1)$-triple. Define

$$
e=-(a+b+c)+2 a b c-2 r s t .
$$

Then there exist integers $u, v, w$ such that

$$
a e+1=u^{2}, \quad b e+1=v^{2}, \quad c e+1=w^{2}
$$

and

$$
c=a+b-e+2(a b e+r u v) .
$$

Proof. This lemma is a special case of Lemma 3 in [14].

Using this lemma we now prove:

Lemma 16. If $v_{m}=w_{n}, n \neq 0,1,2$ and $3 b<c<b^{1.1}$, then $n \geq$ $0.25 \cdot c^{0.2}$.

Proof. With the same notation as in Lemma 14, again we have the congruence

$$
2\left(m^{2}-b n^{2}\right) \equiv \mp A n(\bmod c)
$$

In this case we get

$$
A<\frac{c^{2}+4 b^{2} c}{4 b c-3 c}=b+\frac{c+3 b}{4 b-3}<b+\frac{2 c}{3 b} .
$$

Hence, $A=b+\alpha$, where $0<\alpha<c^{0.1}$.

By Lemma 15, there exist integers $e, u, v, w$ such that

$$
e+1=u^{2}, \quad b e+1=v^{2}, \quad c e+1=w^{2},
$$

and

$$
b=1+c-e+2 c e-2 s u w .
$$

Hence, $(b+e-1)^{2} \equiv 4(c-1)(e+1)(c e+1) \equiv-4(e+1)(\bmod c)$, and

$$
b^{2}+2 b(e-1)+e^{2}+2 e+5 \equiv 0(\bmod c) \text {. }
$$


Moreover, $e$ is small compared with $c$. Indeed, from $c=1+b-e+2 b e+2 r u v$ $>2 b e$, we find that $e<c / 2 b<c^{0.1}$.

Now (18) implies

$$
2 m^{2} \pm \alpha n \equiv b\left(2 n^{2} \mp n\right)(\bmod c) .
$$

From (20), we also get

$$
\left(2 m^{2} \pm \alpha n\right)^{2} \equiv b^{2}\left(2 n^{2} \mp n\right)^{2}(\bmod c)
$$

and

$$
2(e-1)\left(2 n^{2} \mp n\right)\left(2 m^{2} \pm \alpha n\right) \equiv 2(e-1) b\left(2 n^{2} \mp n\right)^{2}(\bmod c) .
$$

By summing (21) and (22), and taking into account (19), we obtain

$$
\begin{aligned}
\left(2 n^{2} \mp n\right)^{2}\left(e^{2}+2 e+5\right) & +\left(2 m^{2} \pm \alpha n\right)^{2} \\
+ & 2(e-1)\left(2 n^{2} \mp n\right)\left(2 m^{2} \pm \alpha n\right) \equiv 0(\bmod c) .
\end{aligned}
$$

Assume now that $n<0.25 \cdot c^{0.2}$. Since $n \geq 3, e \geq 3, m \leq 2 n$ and $c \geq 82$, we have the following estimates:

$$
\begin{gathered}
\left|\left(2 n^{2} \mp n\right)^{2}\left(e^{2}+2 e+5\right)\right| \leq 6 n^{4} \cdot 3 e^{2} \cdot \frac{18 c^{0.8}}{256} \cdot \frac{c^{2}}{4 b^{2}}<0.02 c, \\
\left(2 m^{2} \pm \alpha n\right)^{2} \leq 8 m^{4}+\alpha^{2} n^{2} \leq \frac{c^{0.8}}{2}+\frac{c^{0.6}}{8}<0.23 c
\end{gathered}
$$

and

$$
\left|2(e-1)\left(2 n^{2} \mp n\right)\left(2 m^{2} \pm \alpha n\right)\right| \leq 6 e n^{2}\left(2 m^{2}+\alpha n\right) \leq \frac{3 c^{0.5}}{8} \frac{c^{0.4}}{2}<0.13 c .
$$

Therefore, we have equality in (23), i.e.

$$
\left(e^{2}+2 e+5\right) X^{2}+2(e-1)(e-1) X Y+Y^{2}=0,
$$

where $X=\left(2 n^{2} \mp n\right), Y=2 m^{2} \pm \alpha n$. Since the discriminant of (24) is negative (it is equal to $-16 u^{2}$ ), we conclude that by multiplying (24) with $4\left(e^{2}+2 e+5\right)$ we get

$$
\left(2\left(e^{2}+2 e+5\right) X+2(e-1)^{2} Y\right)^{2}+16 u^{2} Y^{2}=0 .
$$

This implies $X=Y=0$, which in turn gives $n=0$, a contradiction. Hence, we have proved that $n \geq 0.25 \cdot c^{0.2}$.

Again by using Lemma 12 we deduce that $c$ is bounded by an absolute constant.

Proposition 4. Let $\{1, b, c, d\}$ with $1<b<c<d$ be a $D(-1)$ quadruple. If $3 b<c<b^{1.1}$, then $c<0.78 \cdot 10^{94}$ and $d<10^{10^{21}}$.

Proof. Since $b<c / 3$, we have $\log (2.02 b c) \leq 2 \log (0.83 c)<2 \log c$ and $\log (b c) \leq 2 \log (c / 9)$. The inequality $c \leq 1024 n^{5}$ (cf. Lemma 16) and Lem- 
ma 12 imply that

$$
\frac{n-1}{\log (320.72 n)}<0.66 \cdot 10^{12} \cdot \log \left(1024 n^{5}\right) \log \left(113.78 n^{5}\right) .
$$

Hence, $n \leq 0.15 \cdot 10^{19}$ and $c \leq 0.78 \cdot 10^{94}$.

By Lemma 3 we get $\log _{10} d \leq 2 n \log _{10} 16+(4 n+1) \log _{10} c \leq 0.57 \cdot 10^{21}$ and therefore $d \leq 10^{10^{21}}$.

It remains to prove the smallest possible case, i.e. when $c<3 b$. This will be done in the following section.

6. Very small solutions. Now we consider the case $c=1+b+2 \sqrt{b-1}$ of very small solutions (part (vi) in Theorem 1). Observe that we are left with $c \leq 3 b$. By the gap principle (Lemma 4), this implies that we have indeed $c=1+b+2 r$. We can write everything parametrized in $r$. To avoid confusion, we set $k=r$ in this section. Then we have to consider the $D(-1)$-triple

$$
\left\{1, k^{2}+1,(k+1)^{2}+1\right\} \text {. }
$$

Moreover, we have

$$
s=k+1, \quad t=k^{2}+k+1, \quad z_{0}=z_{1}=k+1, \quad x_{0}=0, \quad y_{1}= \pm k,
$$

and $k \geq 8$.

If $v_{m}=w_{n}, n \neq 0,1$ then the congruence from Lemma 2(iii) has the form

$$
\begin{aligned}
m^{2}(k+1) & \equiv n^{2}\left(k^{2}+1\right)(k+1) \pm n\left(k^{2}+k+1\right) k \\
& \equiv n^{2}(k+3) \mp n(k+1)\left(\bmod \left(k^{2}+2 k+2\right)\right) .
\end{aligned}
$$

We start by proving an upper bound for $k$ in terms of $n$.

LEMMA 17. In this case, if $v_{m}=w_{n}, n \neq 0,1$, then $k<4 n^{2}$.

Proof. Assume that $n^{2} \leq \frac{1}{4} k$. We have the estimates

$$
\begin{gathered}
m^{2}(k+1) \leq 4 n^{2}(k+1)<k(k+1)<k^{2}+2 k+2, \\
\left|n^{2}(k+3) \pm n(k+1)\right|<\frac{1}{4} k(k+3)+\frac{1}{2} \sqrt{k}(k+1)<k^{2}+2 k+2 .
\end{gathered}
$$

Therefore we have equality in (25), which gives

$$
m^{2}(k+1)=n^{2}(k+3) \mp n(k+1) .
$$

This implies $m^{2} \equiv 3 n^{2} \mp n(\bmod k)$. Since $m^{2} \leq 4 n^{2}<k$ and $\left|3 n^{2} \mp n\right|<$ $\frac{3}{4} k+\frac{1}{2} \sqrt{k}<k$, we again have equality. This implies $m^{2}=3 n^{2} \mp n$. Moreover, we now also have the equality $m^{2}=n^{2} \mp n$. Combining these two facts, we get $2 n^{2}= \pm n$ and therefore $n=0$, a contradiction. 
We conclude the proof by combining Lemma 17 with Lemma 12 as in all cases above, to get an effective upper bound for $k$ and therefore for $c$ and $d$.

Proposition 5. Let $\left\{1, k^{2}+1,(k+1)^{2}+1, d\right\}$ be a $D(-1)$-quadruple. Then $k<0.14 \cdot 10^{38}$ and therefore $c=(k+1)^{2}+1<0.19 \cdot 10^{75}$ and $d<10^{10^{21}}$.

Proof. We have $\log (2.02 b c)=\log \left(2.02\left(k^{2}+1\right)\left(k^{2}+2 k+2\right)\right) \leq 4.47 \log k$ and $\log \left(k^{2}+2 k+2\right)>\log k$. By combining Lemmas 17 and 12 we get

$$
\begin{aligned}
\frac{n-1}{\log (716.81 n)}< & 0.33 \cdot 10^{12} \log \left(\left(4 n^{2}+1\right)^{2}+1\right) \\
& \cdot \log \left(\left(\left(4 n^{2}\right)^{2}+1\right)\left(\left(4 n^{2}+1\right)^{2}+1\right)\right) .
\end{aligned}
$$

This implies $n<0.90 \cdot 10^{18}$ and therefore $k<0.33 \cdot 10^{37}$.

Moreover, $c=(k+1)^{2}+1<0.11 \cdot 10^{74}$ and by Lemma 3 we have $\log _{10} d \leq 2 n \log _{10} 16+(4 n+1) \log _{10} c \leq 0.27 \cdot 10^{21}$ and so finally $d<10^{10^{21}}$.

Altogether, by combining Propositions 1-5 above, we get Theorem 1. Theorem 2, as well as Corollaries 1 and 2, are immediate consequences.

Acknowledgements. This paper was partly written in the frame of a joint Austrian-Croatian project supported by the Croatian Ministry of Science, Education and Sports and the Austrian Exchange Service (Nr. 20/2004); the authors would like to thank them for this support. Moreover, the first and second authors were supported by the Ministry of Science, Education and Sports, Republic of Croatia, grants 0037110 and 037-03727812821, the second author also by the Austrian Science Foundation FWF, grant NFN S9611, and the third author by the Austrian Science Foundation FWF, grant J2407-N12.

\section{References}

[1] F. S. Abu Muriefah and A. Al-Rashed, On the extendibility of the Diophantine triple $\{1,5, c\}$, Int. J. Math. Math. Sci. 2004, no. 33-36, 1737-1746.

[2] A. Baker and H. Davenport, The equations $3 x^{2}-2=y^{2}$ and $8 x^{2}-7=z^{2}$, Quart. J. Math. Oxford Ser. (2) 20 (1969), 129-137.

[3] A. Baker and G. Wüstholz, Logarithmic forms and group varieties, J. Reine Angew. Math. 442 (1993), 19-62.

[4] M. A. Bennett, On the number of solutions of simultaneous Pell equations, ibid. 498 (1998), 173-199.

[5] E. Brown, Sets in which $x y+k$ is always a square, Math. Comp. 45 (1985), 613-620.

[6] L. E. Dickson, History of the Theory of Numbers, Vol. 2, Chelsea, New York, 1966, 513-520.

[7] Diophantus of Alexandria, Arithmetics and the Book of Polygonal Numbers, I. G. Bashmakova (ed.), Nauka, Moscow, 1974 (in Russian).

[8] —, Die Arithmetik und die Schrift der Polygonalzahlen, G. Wertheim (ed.), Teubner, Leipzig, 1890. 
[9] A. Dujella, Generalization of a problem of Diophantus, Acta Arith. 65 (1993), $15-27$.

[10] -, On the exceptional set in the problem of Diophantus and Davenport, in: Application of Fibonacci Numbers, Vol. 7, G. E. Bergum, A. N. Philippou, A. F. Horadam (eds.), Kluwer, Dordrecht, 1998, 69-76.

[11] - Complete solution of a family of simultaneous Pellian equations, Acta Math. Inform. Univ. Ostraviensis 6 (1998), 59-67.

[12] - An absolute upper bound for the size of Diophantine m-tuples, J. Number Theory 89 (2001), 126-150.

[13] - An extension of an old problem of Diophantus and Euler II, Fibonacci Quart. 40 (2002), 118-123.

[14] —, On the size of Diophantine m-tuples, Math. Proc. Cambridge Philos. Soc. 132 (2002), 23-33.

[15] - , There are only finitely many Diophantine quintuples, J. Reine Angew. Math. 566 (2004), 183-214.

[16] - Bounds for the size of sets with the property $D(n)$, Glas. Mat. Ser. III 39 (2004), 199-205.

[17] A. Dujella and C. Fuchs, Complete solution of a problem of Diophantus and Euler, J. London Math. Soc. (2) 71 (2005), 33-52.

[18] A. Dujella and F. Luca, Diophantine m-tuples for primes, Int. Math. Res. Not. 2005, no. 47, 2913-2940.

[19] A. Dujella and A. Pethő, A generalization of a theorem of Baker and Davenport, Quart. J. Math. Oxford Ser. (2) 49 (1998), 291-306.

[20] A. Filipin, Non-extendibility of $D(-1)$-triples of the form $\{1,10, c\}$, Int. J. Math. Math. Sci. 2005, no. 14, 2217-2226.

[21] Y. Fujita, The extensibility of $D(-1)$-triples $\{1, b, c\}$, Publ. Math. Debrecen 70 (2007), 103-117.

[22] P. Gibbs, Some rational Diophantine sextuples, Glas. Mat. Ser. III 41 (2006), 195-203.

[23] H. Gupta and K. Singh, On k-triad sequences, Int. J. Math. Math. Sci. 5 (1985), 799-804.

[24] T. L. Heath, Diophantus of Alexandria: A study in the history of Greek Algebra. With a supplement containing an account of Fermat's theorems and problems connected with Diophantine analysis and some solutions of Diophantine problems by Euler, Cambridge, 1910; Powell's Bookstore, Chicago; Martino Publishing, Mansfield Center, 2003.

[25] K. S. Kedlaya, Solving constrained Pell equations, Math. Comp. 67 (1998), 833-842.

[26] O. Kihel, On the extendibility of the set $\{1,2,5\}$, Fibonacci Quart. 38 (2000), 464-466.

[27] E. M. Matveev, An explicit lower bound for a homogeneous rational linear form in logarithms of algebraic numbers. II, Izv. Ross. Akad. Nauk Ser. Mat. 64 (2000), no. 6, 125-180 (in Russian).

[28] S. P. Mohanty and A. M. S. Ramasamy, The simultaneous Diophantine equations $5 y^{2}-20=x^{2}$ and $2 y^{2}+1=z^{2}$, J. Number Theory 18 (1984), 356-359.

[29] —, - On $P_{r, k}$ sequences, Fibonacci Quart. 23 (1985), 36-44.

[30] J. H. Rickert, Simultaneous rational approximations and related Diophantine equations, Math. Proc. Cambridge Philos. Soc. 113 (1993), 461-472.

[31] P. G. Walsh, On two classes of simultaneous Pell equations with no solutions, Math. Comp. 68 (1999), 385-388. 
[32] D. X. Zheng, On the systems of Diophantine equations $y^{2}-2 x^{2}=1, z^{2}-5 x^{2}=4$ and $y^{2}-5 x^{2}=4, z^{2}-10 x^{2}=9$, Sichuan Daxue Xuebao 24 (1987), 25-29.

Andrej Dujella

Department of Mathematics

University of Zagreb

Bijenička cesta 30

10000 Zagreb, Croatia

E-mail: duje@math.hr
Clemens Fuchs

Department of Mathematics

ETH Zurich

Raemistrasse 101

8092 Zürich, Switzerland

E-mail: clemens.fuchs@math.ethz.ch

Alan Filipin

Faculty of Civil Engineering

University of Zagreb

Fra Andrije Kačića-Miošića 26

10000 Zagreb, Croatia

E-mail: filipin@grad.hr

Current address:

Department for Analysis

and Computational Number Theory

TU Graz

Steyrergasse 30

8010 Graz, Austria

Received on 9.10.2006

and in revised form on 18.4.2007 